\title{
Matrixeffekte bei der pH-Messung von Bodenproben mit Antimonelektroden
}

\author{
Manfred Decker ${ }^{1}$, Susanne Bause ${ }^{1,2}$, Petra Teichmann ${ }^{1}$, Winfried Vonau ${ }^{1}$ \\ ${ }^{1}$ Kurt-Schwabe-Institut für Mess- und Sensortechnik e.V. Meinsberg, Waldheim, Deutschland, \\ ${ }^{2}$ Hochschule Mittweida, University of Applied Sciences, Mittweida, Deutschland \\ Kontakt:decker@ksi-meinsberg.de
}

\begin{abstract}
Zusammenfassung:
Zielsetzung des Vorhabens ist die schnelle Messung des pH-Wertes von Ackerböden vor Ort angelehnt an die Vorschriften der DIN ISO 10390. Zur pH-Messung in aufgeschlämmten Ackerbodenproben bietet sich alternativ zur zerbrechlichen Glaselektrode eine Antimonelektrode an. Die für dies Vorhaben am KSI gefertigte Elektrode zeichnet sich durch eine gute $\mathrm{pH}$-Sensitivität im relevanten $\mathrm{pH}$ Bereich von 4 bis 9 aus. Untersuchungen von Aufschlämmungen verschiedener Bodentypen in den in der DIN-Norm verwendeten Extraktionslösungen zeigten bei der Verwendung von destilliertem Wasser und 0,01 $\mathrm{M} \mathrm{CaCl}_{2}$-Lösung eine gute Übereinstimmung der Resultate der Antimonelektrode mit denen der im DIN-Verfahren vorgeschriebenen pH-Glaselektrode. Zur Bestimmung von Matrixeffekten wurden zu pH-Pufferlösungen definierte Mengen an potenziell interferierenden Substanzen gegeben. Huminsäure-Suspensionen und gesättigte Tannin- und Lignin-Lösungen übten nahezu keinen Einfluss auf das Elektrodenpotential aus, während die Addition von Gallussäure zu deutlichen Änderungen führte.
\end{abstract}

Schlüsselwörter: Antimonelektrode, pH-Messung von Ackerböden, vor-Ort-Messung, Matrixeffekte, DIN ISO 10390

\section{Zielsetzung}

Für den Landwirt ist die pH-Verteilung auf seinen Ackerböden ein wichtiges Kriterium zur Beurteilung der Qualität der Nutzflächen. Aufbauend auf diesen Resultaten kann er die Dosierung des Düngemitteleinsatzes steuern, den Ernteertrag erhöhen und Kosten senken. Mittelfristiges Ziel der vorgestellten Untersuchungen ist die Realisierung eines automatisierten Systems zur Probennahme und $\mathrm{pH}$ Messung. Dadurch soll eine schnelle Kartographierung der $\mathrm{pH}$-Verteilung schon vor Ort ermöglicht werden, die dann als fundierte Basis für eine ortsaufgelöste Düngung dient. Bei der Realisierung der technischen Herausforderung soll auf die robuste Antimonelektrode zur $\mathrm{pH}$ Messung zurückgegriffen werden, da die mechanisch sehr zerbrechliche pH-Glaselektrode unter den harschen Arbeitsbedingungen auf landwirtschaftlichen Ackerflächen in den abrasiven Medien ein hohes Ausfallrisiko aufweist.

\section{Stand der Technik}

Die pH-Messung von Bodenproben wird in der DIN ISO 10390 beschrieben [1]. Nach der Probennahme werden die zu untersuchenden
Böden bei max. $40{ }^{\circ} \mathrm{C}$ getrocknet und Grobbestandteile mittels eines Siebs mit Öffnungen von $2 \mathrm{~mm}$ abgetrennt. Als repräsentative Untersuchungsprobe werden von der Probe $5 \mathrm{~mL}$ mit einem Messlöffel entnommen und mit dem fünffachen Volumen einer Extraktionslösung versehen. Dabei können sowohl eine 1 molare Kaliumchloridlösung, eine 0,01 molare Calciumchloridlösung wie auch destilliertes Wasser verwendet werden. Nach einer einstündigen $( \pm 10$ Minuten) innigen Durchmischung der suspendierten Probe wird anschließend mittels einer Glaselektrode das Potential ermittelt und der $\mathrm{pH}-$ Wert errechnet.

Von der Probennahme bis zur Übermittlung des Messergebnisses vergeht i.d.R. ein längerer Zeitraum, so dass eine zeitnahe Düngung für den Landwirt nicht mehr möglich ist. Darüber hinaus erlauben die Kosten für die notwendigen Laboranalysen keine hohe räumliche Auflösung bei der Erstellung des pH-Rasters des beprobten Ackers.

\section{Antimonelektrode}

Schon in den 1920er Jahren wurde der Einsatz der Antimonelektrode bei der pH-WertBestimmung beschrieben [2-4]. Es handelt sich bei diesem Messfühler um eine sogenannte 
Elektrode zweiter Art, bei der das auf der Oberfläche des Antimons vorhandene und ständig neu gebildete $\mathrm{Sb}(\mathrm{OH})_{3}$ die $\mathrm{pH}$-Wertabhängige Potentialeinstellung bewirkt [5]. Von Sensoren dieses Typs kann ein $\mathrm{pH}$-Bereich von ca. 3 bis 11 abgedeckt werden [6]. Treibende Kraft bei der Entwicklung der Antimonelektrode war die Suche nach robusten Alternativen zur fragilen $\mathrm{pH}$-Glaselektrode. So wurde dieser Sensor schon im Jahr 1928 zur Bestimmung des $\mathrm{pH}$-Wertes von Bodenproben herangezogen. Im Rahmen der vorgestellten Untersuchungen kamen am KSI hergestellte Metallelektroden mit einem aus massivem Antimon gebildeten Sensorkopf zum Einsatz. Die Abbildung 1 zeigt eine der verwendeten Antimonelektroden. Der sensitive Metallkern ist an der Spitze des Edelstahlkörpers platziert.

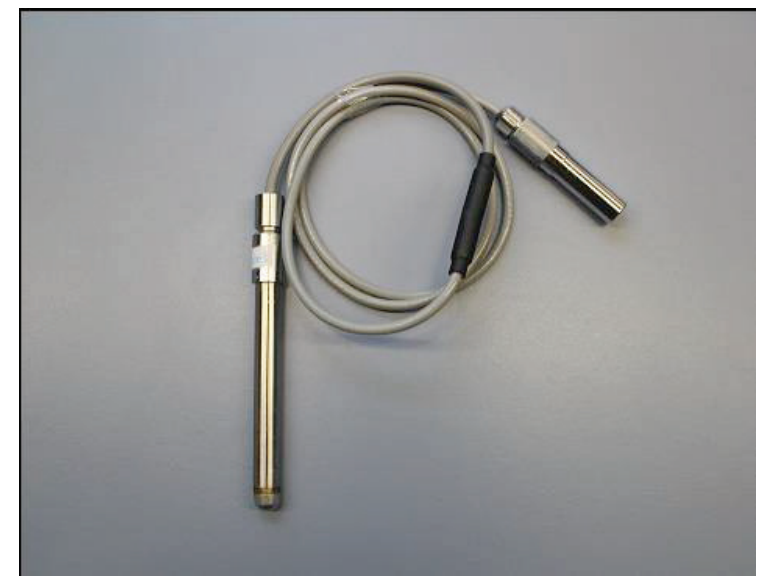

Abb. 1. Am KSI gefertigte $\mathrm{pH}$-sensitive Antimonelektrode.

\section{Material und Methoden}

Für die Untersuchungen wurden Chemikalien des Reinheitsgrades p.a. verwendet und die Lösungen jeweils frisch mit destilliertem Wasser hergestellt. Die verwendete Tannin- und LigninLösung war ein Produkt der Firma Sigma Aldrich Chemie $\mathrm{GmbH}$, Taufkirchen. Bei der Huminsäure und der Gallussäure wurde auf Substanzen der Carl Roth $\mathrm{GmbH}$ \& Co. KG, Karlsruhe, zurückgegriffen. Die pH-Messungen der verschiedenen Bodenproben wurden entsprechend den Vorschriften der DIN-Norm [1] ausgeführt. Die Aufzeichnung der Potentiale der Antimon- wie auch der $\mathrm{pH}$-Glaselektroden (ebenfalls eine Eigenfertigung des $\mathrm{KSI}$ ) erfolgte mit einem pH-Meter Multi-Calimatic 764 der Fa. Knick in Kombination mit einer gesättigten $\mathrm{Ag} / \mathrm{AgCl}$-Referenzelektrode (SSE).

\section{Messergebnisse und Resultate}

Der Abbildung 2 zeigt, dass die Antimon- und die $\mathrm{pH}$-Glaselektrode ein vergleichbar schnelles Ansprechen beim Wechsel der Pufferlösungen aufweisen.

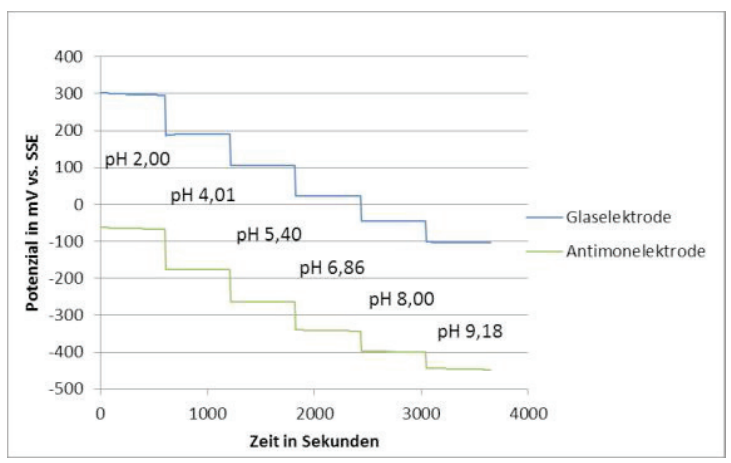

Abb. 2. Ansprechverhalten einer Antimonelektrode und einer $\mathrm{pH}$-Glaselektrode in verschiedenen Pufferlösungen; Temperatur $25^{\circ} \mathrm{C}$.

In Abbildung 3 sind Kalibrierkurven für beide Elektrodentypen aufgeführt. Bei der Antimonelektrode ist im alkalischen Bereich eine Abnahme der Sensitivität um ca. $25 \%$ gegenüber der Kennlinie im sauren Bereich zu erkennen. Die Verringerung der Elektrodensteilheit kann auf den Bereich zwischen $\mathrm{pH}$ Werten 6,8 und 7,1 lokalisiert werden.

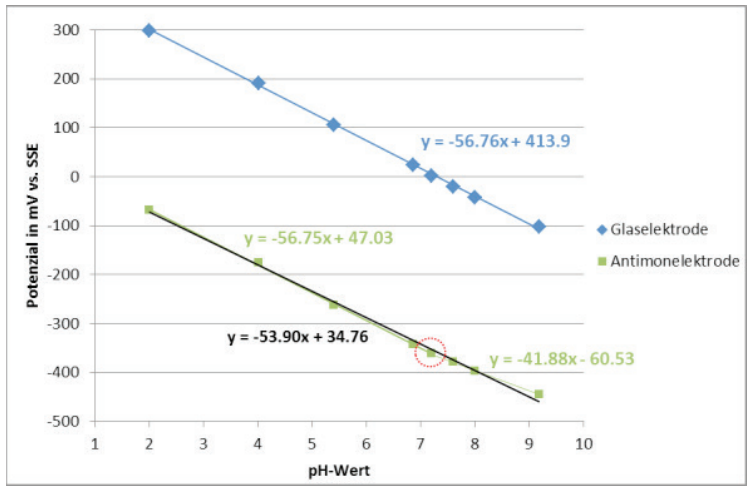

Abb. 3. Kalibrierfunktionen einer Antimonelektrode und einer $\mathrm{pH}$-Glaselektrode im Bereich pH 2 - 9; bei der Metallelektrode sind zusätzlich noch die Kalibrierfunktionen für die $\mathrm{pH}$-Werte von 2-7 und 7-9 eingefügt; Temperatur $=25^{\circ} \mathrm{C}$.

Anschließende pH-Messungen erfolgten in Aufschlämmungen von verschiedenen Bodenproben, die nach den Vorschriften von [1] aufbereitet wurden. Beispielhaft wurden folgende Bodenqualitäten ausgewählt:

Boden 1: schwach toniger Schluff

Boden 2: lehmiger Ton

Boden 3: Sand 
Als Extraktionsmittel dienten destilliertes Wasser, 1 molare $\mathrm{KCl}$-Lösung und 0,01 molare $\mathrm{CaCl}_{2}$-Lösung. Die Unterschiede der $\mathrm{pH}$-Resultate bei Verwendung der verschiedenen Elektroden sind der folgenden Tabelle $1 \mathrm{zu}$ entnehmen.

Tab. 1: Vergleich der pH-Messungen mit einer Glaselektrode und einer Antimonelektrode in verschiedenen Bodenproben (Messvorbereitung analog DIN ISO 10390).

\begin{tabular}{|l|l|l|}
\hline $\begin{array}{l}\text { Boden/Extraktions- } \\
\text { mittel }\end{array}$ & $\begin{array}{l}\text { pH-Glas- } \\
\text { elektrode }\end{array}$ & $\begin{array}{l}\text { Antimon- } \\
\text { elektrode }\end{array}$ \\
\hline $1+$ dest. Wasser & 5,66 & 5,62 \\
\hline $1+1 \mathrm{M} \mathrm{KCl}$ & 4,72 & 4,84 \\
\hline $1+0,01 \mathrm{M} \mathrm{CaCl}_{2}$ & 4,96 & 5,00 \\
\hline $2+$ dest. Wasser & 8,08 & 7,83 \\
\hline $2+1 \mathrm{M} \mathrm{KCl}$ & 7,35 & 7,05 \\
\hline $2+0,01 \mathrm{M} \mathrm{CaCl}_{2}$ & 7,52 & 7,25 \\
\hline $3+$ dest. Wasser & 5,61 & 5,64 \\
\hline $3+1 \mathrm{M} \mathrm{KCl}$ & 4,29 & 4,55 \\
\hline $3+0,01 \mathrm{M} \mathrm{CaCl}$ & 4,64 & 4,57 \\
\hline
\end{tabular}

Die Resultate der Messungen zeigen die Eignung der vom KSI gefertigten Antimonelektrode zur Bestimmung des $\mathrm{pH}$-Wertes in den Aufschlämmungen der Bodenproben auf. Allerdings sind die Abweichungen bei den Messungen Bodenproben suspendiert in 1 molarer $\mathrm{KCl}$-Lösung insbesondere im sauren Bereich deutlich höher als bei der Verwendung von destilliertem Wasser oder 0,01 molarer $\mathrm{CaCl}_{2}$-Lösung als Extraktionsmittel.

Die Antimonelektrode zeichnet sich in den Aufschlämmungen der Bodenproben durch ein schnelles Einstellverhalten aus. In Abbildung 4 ist dies exemplarisch für Boden 1 aufgezeigt.

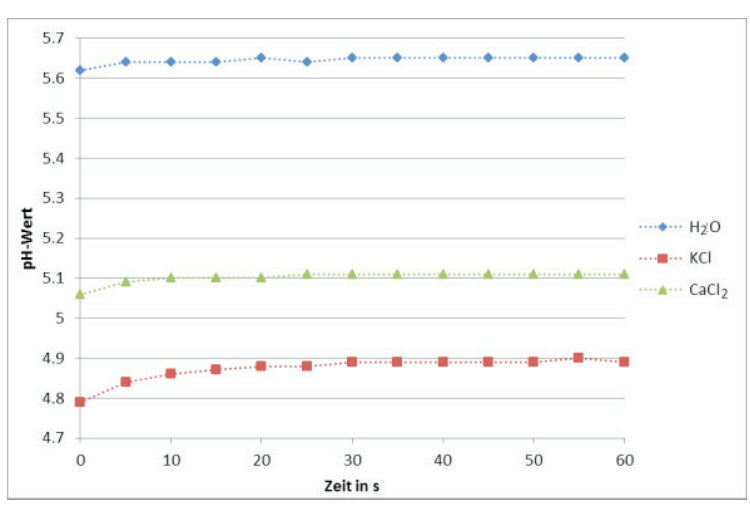

Abb. 4. Ansprechverhalten der Antimonelektrode in der ersten Minute in Aufschlämmungen der Bodenprobe 1 in Extraktionsmitteln nach [1].
Definierte Mengen der Bodenprobe 1 wurden dazu in verschiedenen Extraktionslösungen suspendiert und die Potentialbildung registriert. Schon nach 30 Sekunden ist ein konstanter $\mathrm{pH}-$ Wert erreicht, wie es in der Praxis auf dem Feld zur Gewährleistung einer hohen Beprobungszahl und -dichte erforderlich ist.

Weitere Messreihen dienten der Untersuchung potenzieller Matrixeffekte verschiedener organischer Bodeninhaltsstoffe auf die pHMessung mit der Antimonelektrode. Als Störsubstanzen wurden käufliche Huminsäure sowie Lignin- und Tannin-Lösung verwendet. Die Aufschlämmung mit Huminsäure, bzw. die reine Ausgangslösung (Tannin und Lignin) wurden definiert (1/10 des Vorlagevolumens) zu den Puffer-Lösungen gegeben und die Potentialänderungen mit der Antimonelektrode verfolgt. Durch die Zugaben hervorgerufene $\mathrm{pH}-$ Wert-Änderungen wurden parallel mit einer Glaselektrode registriert.

In Abbildung 5 ist der Einfluss der Zugabe einer gesättigten Tannin- und Lignin-Lösung auf die Potentiallagen der Antimon- und der $\mathrm{pH}-\mathrm{Glas}-$ elektrode bei verschiedenen $\mathrm{pH}-$ Werten zu erkennen. Die Änderungen betragen bei allen vorgelegten Puffern weniger als $+3,5 \mathrm{mV}$. Diese Abweichung kann im Rahmen eines Feldeinsatzes der $\mathrm{pH}$-Sensoren vernachlässigt werden. Vergleichbare Resultate wurden bei der Zugabe von Aufschlämmungen von käuflicher Huminsäure erhalten.

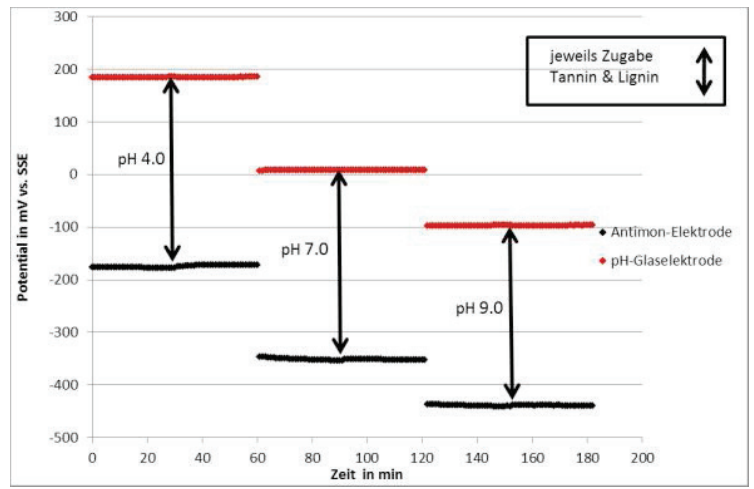

Abb. 5. Vergleich der Potentialänderungen einer Antimonelektrode und einer $\mathrm{pH}$-Glaselektrode auf die Zugabe einer gesättigten Tannin- und Lignin-Lösung (1/10 des Vorlagevolumens) zu verschiedenen $\mathrm{pH}$ Pufferlösungen.

Darüber hinaus wurde exemplarisch der Einfluss von reiner Gallussäure, einem antioxidativ wirkenden Baustein zahlreicher im Boden enthaltener Gerbstoffe, auf die Potentiallage der Antimonelektrode untersucht. Der Abbildung 6 können die Potentialänderungen in verschiedenen Pufferlösungen 
nach Zugabe von Gallussäure-Lösungen entnommen werden.

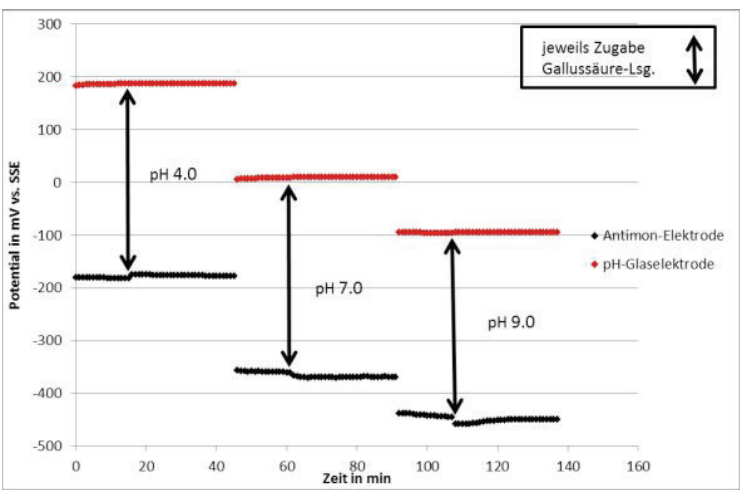

Abb. 6. Vergleich der Potentialänderungen einer Antimonelektrode und einer $\mathrm{pH}$-Glaselektrode auf die Zugabe von Gallussäure-Lösungen (1/10 des Vorlagevolumens, Gehalt Gallussäure $1 \mathrm{~g} / \mathrm{L}$ ) $z u$ verschiedenen Pufferlösungen.

Bei der gewählten Konzentration (1 $\mathrm{g} / \mathrm{L})$ an Gallussäure kann der Einfluss auf die Potentiallage nicht vernachlässigt werden. Bei $\mathrm{pH}=4,0$ resultieren Potentialänderungen, die einem $\mathrm{pH}-$ Wert von 3,8 entsprechen. Bei der Vorlage der Puffer pH 7,0 und 9,0 werden nach Zugabe der Verbindung hingegen Potentiale ermittelt, die zu einer rechnerischen Verschiebung der $\mathrm{pH}$-Werte um 0,3 Einheiten in den alkalischen Bereich führen würden. Auch wenn die in den Untersuchungen verwendete Konzentration der Gallussäure deutlich über den in der Natur zu erwartenden Werten liegt, wird zur Abschätzung weiterer potenzieller Interferenzen in weiterführenden Messreihen zu klären sein, ob die Effekte auf Wechselwirkung der Gallussäure mit den Oberflächenoxiden des Antimons, auf Änderungen des Redoxpotentials oder auf Reaktionen des Antioxidants mit dem gelösten Sauerstoff und daraus resultierenden Einflüssen auf die Bildung der Oberflächenoxide zurückzuführen sind.

\section{Diskussion}

Die bisherigen Messergebnisse zeigen, dass die vom KSI hergestellte robuste Antimonelektrode mit massivem Sensorkopf zur Messung des $\mathrm{pH}-$ Wertes in aufgeschlämmten Bodenproben geeignet ist. Von den in [1] vorgeschriebenen Extraktionsmitteln zeigen die Elektroden in Aufschlämmungen in destilliertem Wasser und in 0,01 molarer $\mathrm{CaCl}_{2}$-Lösung die besten Resultate, während bei der Verwendung einer 1 molaren $\mathrm{KCl}$-Lösung etwas größere Matrixeffekte erhalten werden. Die Zugabe von Huminsäure-Aufschlämmungen, sowie Tanninund Lignin-Lösung rufen keine deutlichen Potentialänderungen bei der pH-Messung hervor. Im Gegensatz dazu sind nach der Addition von Gallussäure mit Konzentrationen von $1 \mathrm{~g} / \mathrm{L}$ Potentialabweichungen in den Puffern von bis zu 0,3 pH-Einheiten zu beobachten. Eine tiefergreifende Aufklärung dieses Effekts wird in weiterführenden Arbeiten noch erfolgen müssen.

\section{Ausblick}

Bei der weiteren Bearbeitung des Projektes werden in zusätzlichen Messreihen die Elektroden in Aufschlämmungen mit niedrigeren Anteilen an Extraktionsmitteln untersucht werden. Nur so kann in der Praxis ein hoher Probendurchsatz ohne großen Chemikalienverbrauch bei den Messungen auf dem Acker realisiert werden. Weitere Untersuchungen betreffen die Ermittlung der Temperaturabhängigkeit der Sensorfunktion, um eine geeignete Temperaturkompensation durchzuführen.

Die bisherigen Resultate zeigen deutlich, dass sich die Antimonelektrode als $\mathrm{pH}$-Sensor zur automatisierten Messung von aufgeschlämmten Bodenproben vor Ort auf dem Feld anbietet.

\section{Danksagung}

Die vorgestellten Untersuchungen wurden freundlicherweise von der AiF unter dem Förderkennzeichen KF2218315NT3 unterstützt.

\section{Literatur}

[1] DIN ISO 10390 Bodenbeschaffenheit Bestimmung des pH-Wertes. Ausgabe 2005-12. Beuth-Verlag, 2005

[2] UHL, A. ; KESTRANEK, W.: Die elektrometrische Titration von Säuren und Basen mit der AntimonIndikatorelektrode. In: Monatsh. Chem. 44 (1923), Nr. 1, S. 29-34

[3] KOLTHOFF, I.M., HARTONG, B.D.: The antimony electrode as an indicator for hydrogen ions and its application in potentiometric titrations of acids and bases. In: Recl. Trav. Chim. Pay-B. 44 (1925), Nr. 2, S. 113-120

[4] FRANKE, K.W., WILLAMAN, J.J.: Measurement of hydrogen-ion concentration in the control of pulp and paper manufacture. In: Ind. Eng. Chem. 20 (1928), Nr. 1, S. 87-95

[5] ROBERTS, E.J., FENWICK, F.: The antimonyantimony trioxide electrode and its use as a measure of acidity. In: J. Am. Chem. Soc. 50 (1928), Nr. 8, S. 2125-2147

[6] BATES, R.G.: Electrodes for pH measurement. In: J. Electroanal. Chem. 2 (1961), Nr. 2, S. 93109 